\title{
The dynamic speckle-based wavemeter
}

\author{
Hanson, Steen Grüner; Jakobsen, Michael Linde; Chakrabarti, Maumita
}

\section{Published in:}

Proceedings of SPIE

Link to article, DOI:

$10.1117 / 12.2319873$

Publication date:

2018

Document Version

Publisher's PDF, also known as Version of record

Link back to DTU Orbit

Citation (APA):

Hanson, S. G., Jakobsen, M. L., \& Chakrabarti, M. (2018). The dynamic speckle-based wavemeter. In Proceedings of SPIE (Vol. 10834). [108342D] SPIE - International Society for Optical Engineering. Proceedings of SPIE - The International Society for Optical Engineering https://doi.org/10.1117/12.2319873

\section{General rights}

Copyright and moral rights for the publications made accessible in the public portal are retained by the authors and/or other copyright owners and it is a condition of accessing publications that users recognise and abide by the legal requirements associated with these rights.

- Users may download and print one copy of any publication from the public portal for the purpose of private study or research.

- You may not further distribute the material or use it for any profit-making activity or commercial gain

- You may freely distribute the URL identifying the publication in the public portal 


\section{The dynamic speckle-based wavemeter}

Steen G. Hanson, Michael Linde Jakobsen, Maumita Chakrabarti

Steen G. Hanson, Michael Linde Jakobsen, Maumita Chakrabarti, "The dynamic speckle-based wavemeter," Proc. SPIE 10834, Speckle 2018: VII International Conference on Speckle Metrology, 108342D (7 September 2018); doi: 10.1117/12.2319873

SPIE Event: SPECKLE 2018: VII International Conference on Speckle Metrology, 2018, Janów Podlaski, Poland 


\title{
The dynamic speckle-based wavemeter
}

\author{
Steen G. Hanson, Department of Photonics Engineering, Technical University of Denmark, \\ Denmark $^{1}$ \\ Michael Linde Jakobsen, Department of Photonics Engineering, Technical University of \\ Denmark, Denmark.
}

Maumita Chakrabarti, DenseLight Semiconductor Pte. Ltd. Singapore.

\begin{abstract}
Based on a previously devised speckle-based set-up for probing minute wavelength changes for a coherent field [1], [2] we will here present the first experiments where these changes are resolved on a millisecond time scale. The setup is based on probing the lateral shift of a speckle pattern arising from a slanted rough object, the speckle displacement being linearly proportional to the wavenumber change. Thus, by shearing the speckle pattern across a grating-like structure [3],[4] and [5], a frequency proportional to the frequency of the wavelength change can be derived as will the irradiance. Thus, a cordial display of the complex field amplitude may be obtained with a high temporal resolution and a reasonable spectral resolution. The spatial filter is here preliminarily implemented by recording the speckle pattern with a CMOS array with subsequent digital image processing mimicking the use of a spatial filter.
\end{abstract}

Keywords: Spectrometry, speckle, scattering

\section{Introduction}

Scattering of spatially coherent light from a rough surface will due to constructive and destructive interference give rise to a granular structure, named speckles[6], [7] and [8]. This pattern will appear in case of free space propagation of the scattered field, and usually as well when an optical system transforms the field. The advantage of this pattern with respect to measurement systems is the modulation depth of the intensity, facilitating a precise determination of any movement of the speckles. An abundance of measurement systems have been established based on this fact, e.g. strain measurement [9], surface roughness determination [10] or velocity determination (LDA) [11].

For resolving minute spectral changes for a coherent source, effectively three different approaches have been employed, which will briefly reviewed here:

The first being the grating-based systems relying on diffraction of the incident field from a repetitive structure. Although an abundance of implementations have been put forward, we will here only briefly discuss the ones that possess some of the same features as the one presented in [1] and [2], namely being simple, inexpensive and yet having high resolution. Chaganti et al. [12] offer a miniaturized implementation with a resolution between $0.3 \mathrm{~nm}$ and $4.6 \mathrm{~nm}$ in the visible range, where Kong et al. [13] display a miniaturized IR-spectrometer. These systems are in fact spectrometers that can cope with a continuous spectrum, where the one dealt with in this paper addresses a minute determination of spectral changes for a more or less monochromatic source.

${ }^{1}$ vsgh@fotonik.dtu.dk 
The coherence length of the radiation from a source will reveal its spectral properties. This is employed in the Fourier transform spectrometer, where the incident field is split into two arms of and recombined. Since the travelled distance in the two arms is varied, the modulation depth of the resulting interference pattern will make the correlation function known. The Fourier transform of the modulation depth gives the source's spectral content. A miniaturized version of this imaging system, which calls for rather minute alignment, has been given[14].

The third generic method relies on sending the field into a cavity consisting of two partly reflecting mirrors, and thus establishing a variable bandpass filter by changing he distance between the mirrors. A very sensitive, but delicate, system is established, named a Fabry-Perot interferometer by having two spherical mirrors [15]. A simpler system using fiber optics has been implemented with single mode fibers acting as the two arms [16].

If coherent light is scattered off a rough surface, a change in wavelength may make the speckle pattern shift and/or decorrelate. Two speckle-based system relying on measuring the change/decorrelation of a speckle have been demonstrated; in the first system speckles arise when laser light bounces inside in multimode fiber, at the output of which the speckle pattern will change appearance, uncovering the change in wavelength[17]. Later a second system built around a photonic chip was presented [18]. In both cases, the speckle pattern will change form as a function of the change in wavelength. Therefore, a calibration was needed in order to relate a certain speckle pattern with the corresponding wavelength.

The system, described in the following, will give rise to a linear dependence between speckle displacement and wavelength change.

\section{The speckle-based wavemeter: Theory}

The basic principle for the wavemeter will be superficially presented here, as it was previously shown in [1] and [2]. An incident coherent beam hits the rough surface at an angle $\alpha_{1}$ and the diffusely scattered light is recorded by a detector array placed at an angle $\alpha_{2}$. For simplicity, we assume these two angles to be in the same plane as the normal to the scattering surface. The simple setup is shown in Fig.1.

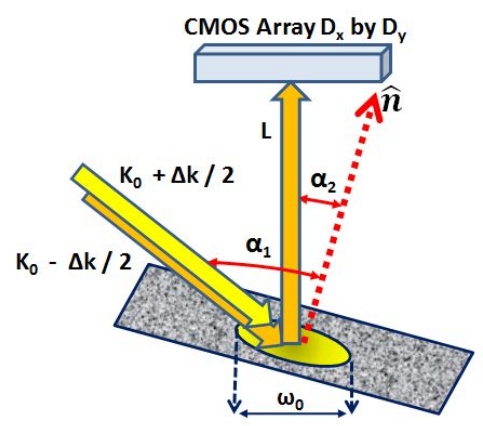

Fig. 1. Simple setup for probing wavelength changes. A change in wavenumber of an incident field $\left(K_{0}-\Delta k / 2\right.$ to $\left.K_{0}+\Delta k / 2\right)$ gives rise to a speckle displacement observed by a CMOS array placed at a distance $L$.

The detector array is placed at a distance $L$, and we treat the scenario, where the wavenumber of the incident field is changed from $K_{0}-\Delta k / 2$ to $K_{0}+\Delta k / 2$. The footprint of the illumination in the plane of detection is $\omega_{0}$. A change in wavenumber will make the speckle pattern shift proportionally due to the linear phase change across the surface.

The crosscovariance $C\left(\mathbf{P}, \Delta \mathbf{p} ; K_{0}, \Delta k\right)$ of the speckle pattern before and after a shift $\Delta \mathbf{p}$ at position $\mathbf{P}$ in wavenumber is defined by 


$$
C\left(\mathbf{P}, \Delta \mathbf{p} ; K_{0}, \Delta k\right)=\left\langle I\left(\mathbf{P}-\frac{\Delta \mathbf{p}}{2} ; K_{0}-\frac{\Delta k}{2}\right) I\left(\mathbf{P}+\frac{\Delta \mathbf{p}}{2} ; K_{0}+\frac{\Delta k}{2}\right)\right\rangle
$$

where we have omitted an unimportant additive background, which is independent on the change in wavenumber. Angular brackets here denote "ensemble average". Assuming the scattering structure to give rise to fully developed speckles, the statistics for the field will be a circular symmetric Gaussian distribution, and therefore the ensemble average over four field components in Eq. 1 can be simplified into the absolute square of the field correlation, which can be easily obtained. Following the derivations from [1] and [2], we arrive at the following expression for the crosscovariance of the speckle pattern before and after a change in wavelength:

$$
C\left(\mathbf{0}, \Delta \mathbf{p} ; K_{0}, \Delta k\right) \propto \exp \left[-\frac{4 R^{2} \cos \left[\alpha_{1}\right]^{2}\left(\Delta p_{x} K_{0} \cos \left[\alpha_{2}\right]-\Delta k L\left(\sin \left[\alpha_{1}\right]+\sin \left[\alpha_{2}\right]\right)\right)^{2}}{\left(\Delta k \omega_{0}(L-R)\right)^{2}+\left(\frac{4 L R}{\omega_{0}} \cos \left[\alpha_{1}\right]^{2}\right)^{2}}\right] .
$$

A finite radius of curvature, $R$, of the incident beam has here been included in the derivation. The sign of $R$ is positive for a converging field. This equation disclose the most important parameters for the setup. We get a shift in position of the speckle pattern:

$$
\Delta p_{x 0}=L \frac{\sin \left[\alpha_{1}\right]+\sin \left[\alpha_{2}\right]}{\cos \left[\alpha_{2}\right]} \frac{\Delta k}{K_{0}}
$$

a speckle size:

$$
\rho_{0}=\frac{2 L \cos \left[\alpha_{1}\right]}{K_{0} \omega_{0} \cos \left[\alpha_{2}\right]}
$$

and a decorrelation depending on the change in wavenumber given by:

$$
\Delta k_{\text {decorr }}=\left|\frac{4 L R \cos \left[\alpha_{1}\right]^{2}}{\omega_{0}^{2}(L-R)}\right|
$$

The relation between speckle shift and change in wavenumber given by Eq. 1, shows an interesting and surprising feature, namely that the gearing (ratio between speckle shift and change in wavenumber) can be arbitrarily large as $\alpha_{2}$ approaches $\pi / 2$. However, the drawback for such large angles will be a non-linear speckle shift, the cure for which will be discussed in the next section.

A good estimate of the spectral resolution is given by the accuracy with which the center of the crosscovariance can be estimated. A good estimate for this is the width of the crosscovariance peak divided by the square root of the number of speckles at the detector array. Following Ref. [2], we get the spectral resolution to be given by

$$
\frac{\Delta k}{K_{0}} \cong \frac{0.28 \lambda}{\sqrt{N_{p i x}} \omega_{0} \tan \left[\alpha_{0}\right]},
$$

where we have assumed $\alpha_{1}=\alpha_{2}=\alpha_{0}$, the number of pixels in the array being $N_{p i x}$ and finally having assumed the speckle size to be three pixels. This gives a spectral resolution of $\Delta k / K_{0}=2.7 \cdot 10^{-6}$. We have here assumed a 
wavelength of $760 \mathrm{~nm}$, a spot size on the target of $1 \mathrm{~mm}$ and a linear array with 1000 pixels. This corresponds to a resolution of approximately $1 \mathrm{GHz}$. If we take advantage of a 2D array, and assume we only have 100 pixels perpendicular to the direction of speckle shift, i.e. the y-direction, the resolution would become $100 \mathrm{MHz}$.

\section{The speckle-based wavemeter: Dynamic measurements}

The previous discussion on spectral resolution relied on recording of a speckle pattern before and after a change in wavenumber, thus limiting the temporal resolution. Next, we investigate the possibilities for simultaneous measurement of irradiance and wavenumber with a high temporal resolution. Spatial filtering velocimetry, cf. [3] and [5], will form the basis for doing this. This method has previously been used for probing jittering of speckles arising from in-plane vibrations of a solid surface [4]. If a speckle pattern is "sheared" across an absorption grating structure with a pitch $\Lambda$, i.e. the period of the grating, the transmitted light is modulated with a frequency given by the speckle velocity divided by the pitch. Only the velocity component perpendicular to the grating structure is measured. Furthermore, the direction of the velocity is not revealed by the simple version. However, the quadrature component of the measured oscillation will tell the direction of the speckle movement. As the frequency is proportional to the speckle velocity, the number of periods or fraction of a period for the oscillation gives the speckle displacement and thus the change in frequency.

In order to verify the possibility of using Spatial Filtering Velocimetry we used speckle images obtained while varying the temperature of the coherent source, here a Vertical Cavity Surface Emitting Laser (VCSEL, type TO 510). Each of the speckle recordings were multiplied with two grating structures, of which one was offset in phase by $\pi / 2$ thus giving the quadrature component. Figure 2 shows an example of a speckle pattern and one of the associated grating structures, numerically applied afterwards to get the frequency.
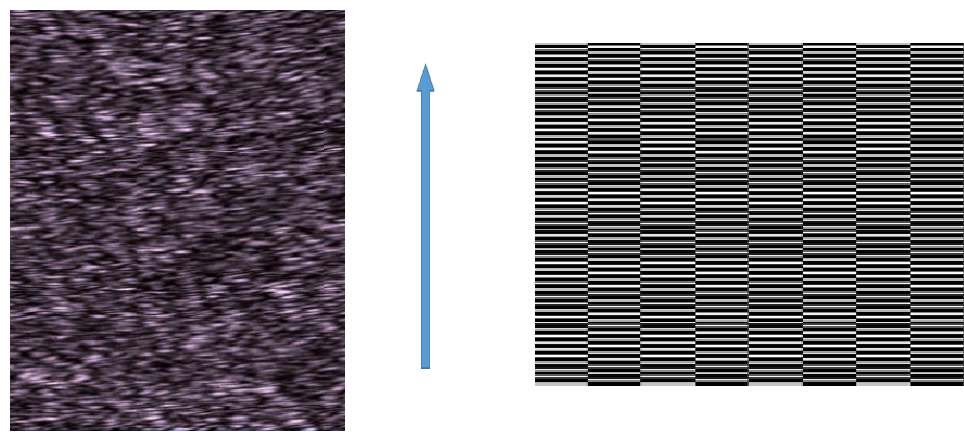

Fig. 2. Example of a speckle pattern, left, and the associated grating structure over which the pattern is sheared. The arrow indicates the speckle displacement when the wavenumber is changed.

A series of speckle patterns (21 ea.) was recorded while varying the temperature of the VCSEL. Subsequently, the real- and imaginary part for each of the speckle patterns were derived. This sequence is shown in Fig. 3a. When the real and imaginary parts are inserted in a polar plot, we get the cordic transformation, where the change in angle from one recording to the next gives the change in wavenumber and the change in length of the phasor provides information on the change in laser power. 


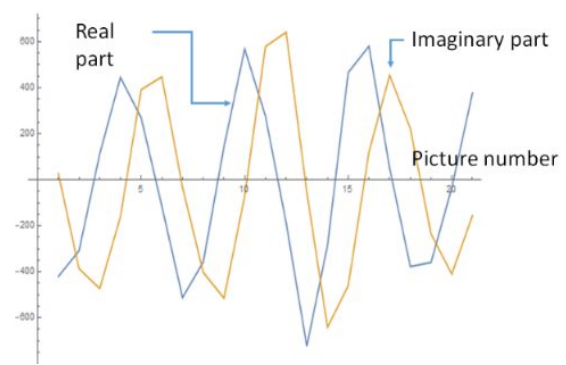

(a)

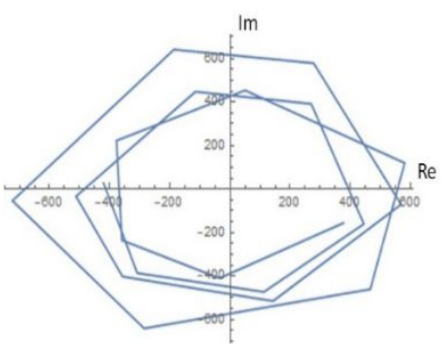

(b)

Fig. 3. The real and imaginary part of each of the 21 speckle patterns, left, and these readings shown as a polar plot to the right.

It is emphasized that the absolute value of the phasor for the speckle pattern (real- versus imaginary part) is of no importance. Only the change in angle and length of the phasor carries information. A one-pitch change of speckle position corresponds to one revolution in the cordic plane (Fig.3b). In our case, the pitch was 20 pixels. Here a displacement of one pixel corresponds to a wavelength change of $11.4 \mathrm{pm}$ giving that one revolution in the cordic corresponds to $229 \mathrm{pm}$.

Using the above method for deriving the change in irradiance and wavenumber, the temporal resolution will be limited due to the update rate of the array detector. An increase of the temporal resolution is obtained by using the methods from Spatial Filtering Velocimetry, [3], [4] and [5]. Alternatively, an array of high-speed single detectors can be arranged and connected to provide the real- and imaginary part of the speckle signal. A higher sensitivity can be achieved by increasing the angle between the normal to the surface and the observation angle, with the drawback of having a variation of the speckle velocity across the grating. This can be circumvented by having a grating with variable pitch.

\section{Conclusions}

The basic principle of the speckle-based wavemeter has been revisited and the first use of Spatial Filtering Velocimetry (SFV) is shown. A series of speckle images obtained for free space propagation of diffusely scattered light from a VCSEL were digitally analyzed as would be the case for SFV processing. A real and imaginary value of a spectral component of the speckle pattern were found, and a complex number was associated to each of the recordings. The change of the argument to this complex number provided the change in wavenumber, and the change in its absolute value revealed the change in irradiance form the scattering surface, and therefore the change in laser power. An experiment demonstrated this, when the temperature of the VCSEL was changed.

\section{References}

1. M. Chakrabarti, M. L. Jakobsen, and S. G. Hanson, "Speckle-based spectrometer," Opt. Lett. 40(14), (2015).

2. S. G. Hanson, M. L. Jakobsen, and M. Chakrabarti, "Speckle-based wavemeter," in SPECKLE 2015: VI International Conference on Speckle Metrology (2015).

3. T. Asakura and Y. Aizu, Spatial Filtering Velocimetry: Fundamentals and Applications (Springer Verlag, 2006).

4. M. L. Jakobsen, H. E. Larsen, S. P. Pedersen, and S. G. Hanson, "Optical spatial filtering velocimetry sensor for real-time in-plane vibration control," Proc. SPIE 6189, 61891A (2006).

5. M. L. Jakobsen and S. G. Hanson, "Lenticular array for spatial filtering velocimetry of laser speckles from solid surfaces," Appl. Opt. 43(24), 4643-4651 (2004). 
6. J. W. Goodman, Speckle Phenomena in Optics: Theory and Applications (Roberts \& Company, 2007).

7. J. W. Goodman, "Statistical properties of laser speckle patterns," in Laser Speckle and Related Phenomena (1975), 6, pp. 9-75.

8. H. M. Pedersen, "Theory of speckle dependence on surface roughness," J. Opt. Soc. Am. 66(11), 1204 (1976).

9. I. Yamaguchi, "Speckle Displacement and Decorrelation in the Diffraction and Image Fields for Small Object Deformation," Opt. Acta Int. J. Opt. 28(10), 1359-1376 (1981).

10. D. Léger, E. Mathieu, and J. C. Perrin, "Optical surface roughness determination using speckle correlation technique.," Appl. Opt. 14, 872-877 (1975).

11. L. E. DRAIN, "Doppler Velocimetry," Laser Focus with Fiberoptic Technol. 16(10), 68- (1980).

12. K. Chaganti, I. Salakhutdinov, I. Avrutsky, and G. W. Auner, "A simple miniature optical spectrometer with a planar waveguide grating coupler in combination with a plano-convex lens.," Opt. Express 14(9), 40644072 (2006).

13. S. H. Kong, D. D. L. Wijngaards, and R. F. Wolffenbuttel, "Infrared micro-spectrometer based on a diffraction grating," Sensors Actuators, A Phys. 92(1-3), 88-95 (2001).

14. M. Pisani and M. Zucco, "Compact imaging spectrometer combining Fourier transform spectroscopy with a Fabry-Perot interferometer.," Opt. Express 17(10), 8319-8331 (2009).

15. M. Hercher, "The spherical mirror fabry-perot interferometer.," Appl. Opt. 7(5), 951-966 (1968).

16. T. Yoshino, K. Kurosawa, K. Itoh, and T. Ose, "Fiber-Optic Fabry-Perot Interferometer and its Sensor Applications," IEEE Trans. Microw. Theory Tech. 30(10), 1612-1621 (1982).

17. B. Redding and H. Cao, "Using a multimode fiber as a high-resolution, low-loss spectrometer," Opt. Lett. 37, 3384 (2012).

18. B. Redding, S. F. Liew, R. Sarma, and H. Cao, "Compact spectrometer based on a disordered photonic chip," Nat. Photonics (2013). 